\title{
Binaral interaction and centrifugal input enhances spatial contrast in olfactory bulb activation
}

\author{
Benjamin H. Singer, ${ }^{1}$ Soyoun $\mathrm{Kim}^{2}$ and Michal Zochowski ${ }^{2,3}$ \\ ${ }^{1}$ Neuroscience Graduate Program, University of Michigan, Ann Arbor, MI, USA \\ 2Department of Physics, University of Michigan, Ann Arbor, MI, USA \\ ${ }^{3}$ Biophysics Research Division, University of Michigan, Ann Arbor, MI, USA
}

Keywords: odors, olfactory bulb, optical recording, Terrapene carolensis, voltage-sensitive dyes

\begin{abstract}
We used paired-pulse odorant stimulation, with a conditioning stimulus delivered either ipsilateral or contralateral to a test stimulus, to unmask the effects of centrifugal feedback on olfactory bulb responses. In reptiles and mammals there are no direct connections between the paired olfactory bulbs, and thus all information transfer between the olfactory bulbs depends on feedback from retrobulbar structures. We measured odor-induced activity in the turtle olfactory bulb using a voltage-sensitive dye and a 464-element photodiode array, which allowed us to monitor the spatial variation in activation of the olfactory bulb. We found that both contralateral and ipsilateral conditioning stimuli evoked long-lasting inhibition of olfactory bulb activation. In contrast to previous studies using local field potential recording to monitor activity at a single site, we found that this inhibition increased contrast in the spatial patterning of activation over the dorsal surface of the olfactory bulb. Inhibition was also increased when different odorants were used as conditioning and test stimuli, suggesting a role for centrifugal feedback in olfactory discrimination. These results highlight the functional importance of centrifugal feedback and information processing in a broadly distributed olfactory network.
\end{abstract}

\section{Introduction}

An olfactory stimulus activates an ensemble of olfactory receptor neurons $(\mathrm{ORN})$ in the olfactory epithelium, each of which expresses a single odorant receptor. Multiple receptor neurons expressing the same odorant receptor project their axons to a small number of glomeruli in the olfactory bulb (OB), where they synapse onto mitral and tufted cells as well as juxtaglomerular interneurons and form a fragmented spatial map of olfactory input (Sullivan et al., 1995; Mori et al., 1999; Meister \& Bonhoeffer, 2001). Odorants bind to many receptor proteins and thus a typical odorant stimulus results in spatially widespread activation of the OB (Friedrich \& Korsching, 1997; Rubin \& Katz, 1999; Johnson \& Leon, 2000; Meister \& Bonhoeffer, 2001; Wachowiak et al., 2002). Input to mitral cells at the glomerulus and subsequent information processing is then subject to modulation by two networks of interneurons: a juxtaglomerular network within the glomerular layer and a granule cell network within the external plexiform layer (EPL; Shepherd et al., 2004).

Interactions within the OB network may refine the spatial representation of odorant information by focusing the diffuse activation of glomerular inputs onto a more restricted set of mitral cell outputs (Friedrich \& Korsching, 1997; Cleland \& Linster, 2005; Cleland \& Sethupathy, 2006). In the visual and auditory systems, spatial representations of sensory information are refined through mechanisms of lateral inhibition (Suga, 1995; Cook \& McReynolds, 1998). In the OB, however, the map of chemotopic information is spatially fragmented, and adjacent glomeruli are not necessarily activated by structurally similar odorants (Urban, 2002; Cleland \& Sethupathy, 2006).

Correspondence: Dr Michal Zochowski, ${ }^{2}$ Department of Physics, as above. E-mail: michalz@umich.edu

Received 5 June 2006, revised 1 November 2006, accepted 3 November 2006
However, the $\mathrm{OB}$ receives extensive centrifugal input which may not be subject to the same topographic constrains as direct projections between glomeruli within the OB (Shepherd et al., 2004). Studies of olfactory learning and paired-pulse stimulation indicate that the temporal structure of $\mathrm{OB}$ activity is modulated on longer timescales, with central feedback probably influencing the interaction of mitral and granule cells (Ravel et al., 2003; Martin et al., 2004b; Zochowski $\&$ Cohen, 2005). These central projections may play an important role in pattern completion and memory-dependent interpretation of odorant input in olfactory perception (Davis, 2004).

Although many studies have examined the influence of centrifugal projections to the OB on a local or single-cell scale (Leveteau et al., 1971; Mori \& Takagi, 1978; Nickell \& Shipley, 1993), none have evaluated their effects on spatial patterning at the population level in vivo or in response to odorant stimuli. Here we exploit the fact that, in reptiles and mammals, there are no monosynaptic connections between the paired OBs (Gamble, 1956; Lohman \& Mentink, 1969; Brunjes et al., 2005). Thus, paired-pulse stimulation with a conditioning stimulus delivered to the naris contralateral to the test stimulus will unmask the influence of projections from retrobulbar structures on the $\mathrm{OB}$ response. We compare changes in the spatial patterning of the $\mathrm{OB}$ due to these centrifugal projections to those elicited by an ipsilateral conditioning stimulus, which conditions the entire olfactory system.

\section{Materials and methods Odorant delivery}

Odorants were delivered using a double-barreled olfactometer (Kauer \& Moulton, 1974; Lam et al., 2000). Carrier air was cleaned and desiccated by passing over activated charcoal. Laboratory air saturated 
with odorant vapor was mixed with carrier air in the inner barrel of the odorant applicator. The flow rate of the carrier air was set to $300 \mathrm{~mL} / \mathrm{min}$ and odorant-saturated air was set to $20 \mathrm{~mL} / \mathrm{min}$, resulting in a $7 \%$ concentration of odorant-saturated air. Between odorant presentations, $1500 \mathrm{~mL} / \mathrm{min}$ suction was applied through the outer barrel of the applicator, preventing the release of odorant. During an odorant pulse, a command pulse to a solenoid valve (South Bend Controls, South Bend, IN, USA) turned off the outer barrel suction, resulting in the release of odorant. This olfactometer has been shown to produce square pulses of odorant which terminate within several milliseconds of the control signal (Lam et al., 2000). When different odorants were released in consecutive pulses, an additional solenoid valve controlled the source of odorant-saturated air to the inner barrel of the applicator. Thus, odorants could be quickly switched during a single imaging trial. Additional control over the delivery of odorant was provided by the placement of a tube at the pharyngeal opening of each nasal cavity (see Surgery, below). The two nasal cavities are isolated from each other by the solid nasal septum. Thus, odorant was selectively drawn through either nasal cavity by intrapharyngeal suction $(300 \mathrm{~mL} / \mathrm{min})$ beginning $500 \mathrm{~ms}$ before each odorant pulse and sustained for at least $1 \mathrm{~s}$ after the odor pulse. This allowed for selective stimulation of either OB. In this study both isoamyl acetate and cineole were used as odorant stimuli (Sigma, St Louis, MO, USA).

\section{Animals}

OB responses to odorant stimulation were measured in 21 box turtles (Terrapene sp.). Of these animals, 18 were prepared for measurements using a voltage-sensitive dye and three were stained with a calciumsensitive dye. The olfactory system of turtles has much the same structure as that of other vertebrates, including mammals (Allison, 1953). Turtles were utilized in these experiments because their OB is an established system for the use of voltage-sensitive dyes to monitor neural activity (Lam et al., 2000; Lam et al., 2003; Zochowski \& Cohen, 2005). In addition, the anatomy of the nasopharynx allows for isolation of the two nasal cavities and administration of nasopharyngeal suction.

\section{Surgery and staining}

Before surgery, turtles were anaesthetized with an injection of the fastacting agent medetomidine $(0.2 \mathrm{mg} / \mathrm{kg}$; Pfizer, New York, NY, USA) followed by immersion in ice for $1 \mathrm{~h}$ preceding and throughout all surgical procedures. A cervical collar was placed to prevent retraction of the head. Topical anaesthetic [20\% benzocaine (Orajel); Del Pharmaceuticals, Farmingdale, NY, USA] was applied around the location of the craniotomy. Local anaesthetic (1\% bupivicaine; Sigma) was infiltrated into cervical muscles and pressure points. A polyvinylchloride tube (outer diameter $1.8 \mathrm{~mm}$, inner diameter $1 \mathrm{~mm}$ ) was inserted into the pharyngeal opening of each nasal cavity and affixed to the bony portion of the maxilla with polyacrylate glue and epoxy putty. The pharyngeal opening around each tube was sealed with alginate caulk (Dentsply, Milford, DE, USA) to limit retronasal spread of odorants and isolate airflow in the nasal cavity from the animal's respiration.

The head position was stabilized for the duration of surgery and imaging in a home-made head holder by the use of nonpuncturing ear bars and by clamping the maxilla to a metal bar. The dorsal surface of both OBs was exposed by craniotomy and removal of the dura mater and arachnoid mater. During surgery, the $\mathrm{OB}$ was irrigated with turtle saline of composition (in $\mathrm{mM}$ ): $\mathrm{NaCl}, 96.5 ; \mathrm{KCl}, 2.6 ; \mathrm{MgCl}_{2}, 2.0$;
$\mathrm{NaHCO}_{3}, 31.5 ; \mathrm{CaCl}_{2}, 4.0$; and dextrose, 10.0 (all from Sigma). The saline was bubbled with $95 \% \mathrm{O}_{2}$ and $5 \% \mathrm{CO}_{2}$, and brought to a $\mathrm{pH}$ of $7.0-7.2$.

\section{Voltage-sensitive dye staining}

The fluorescent styryl dye RH414 $(0.1-1.0 \mathrm{mg} / \mathrm{mL})$ was used to monitor activity in the OB (Grinvald et al., 1994). The dye was applied to the exposed bulb after dissection of the meninges. Bath staining was carried out for $60 \mathrm{~min}$, with changes of the dye solution every $15 \mathrm{~min}$. After staining, the OBs were washed with saline and covered with a 3\% agarose gel (Sigma). A glass coverslip was placed on top of the gel to provide a flat imaging window.

\section{Calcium-sensitive dye staining}

ORNs were labelled with Calcium Green-1 dextran, $10 \mathrm{kDa}$ m.w. (Molecular Probes). A $20 \mathrm{mg} / \mathrm{mL}$ dye solution in $0.1 \mathrm{M} \mathrm{NaCl}$ plus $0.5 \%$ Triton $\mathrm{X}-100$ was instilled into each nasal cavity for $20 \mathrm{~min}$. After staining, the animals were held for 5-7 days to allow anterograde transport of the dye into olfactory nerve terminals before measurements were performed.

After surgery, turtles were warmed to room temperature for $1 \mathrm{~h}$ before imaging. The turtles were locally anaesthetized during the recordings. All experimental procedures were approved by the University Committee for the Use and Care of Animals at the University of Michigan, and were in accordance with Public Health Services Policy on the Care and Use of Animals.

\section{Imaging}

During voltage-sensitive dye measurements the preparation was illuminated using a $100 \mathrm{~W}$ halogen bulb. The incident light was heatfiltered and passed through a $520 \pm 30 \mathrm{~nm}$ incident light filter. The emitted light $>610 \mathrm{~nm}$ was collected by a Macroscope $(4 \times$ magnification, 0.95 f; RedShirt Imaging, LLC, Fairfield, CT, USA) onto a 464element photodiode array (NeuroPDA, RedShirt Imaging). For calcium dye measurements, incident light from a $100 \mathrm{~W}$ halogen bulb was filtered at $480 \pm 30 \mathrm{~nm}$ and fluorescence emission was collected $>530 \mathrm{~nm}$. Each pixel of the photodiode array received light from an $\sim 170 \times 170 \mu \mathrm{m}$ area of the image plane. Data were filtered at acquisition at $0.5-1500 \mathrm{~Hz}$ and sampled at $1.6 \mathrm{kHz}$. While high-pass filtering of the data may have resulted in the loss of very slow components of the descending phase of the DC signal and distorted the measurement of $\mathrm{OB}$ activation following paired-pulse stimulation of a single $\mathrm{OB}$, this artifact would have no effect on the measurement of signals when the conditioning and test stimuli were delivered to contralateral OBs.

\section{Data analysis}

All data were acquired using Neuroplex software (RedShirt Imaging) and then exported for analysis. All analysis was performed using built-in and custom routines in MATLAB (The Mathworks, Natick, MA, USA).

\section{Calcium-sensitive dye responses}

Fractional changes in fluorescence responses $(\Delta \mathrm{F} / \mathrm{F})$ from animals stained with Calcium Green-1 were calculated by dividing the output of the photodiode array by the resting fluorescence observed at each pixel before the presentation of odorant. Responses were then digitally filtered using low-pass Gaussian and high-pass Butterworth filters $(0.1-2.0 \mathrm{~Hz})$. The results in Fig. 1 were obtained from spatial averages over 5-10 pixels, with the same set of pixels used in the analysis of all 

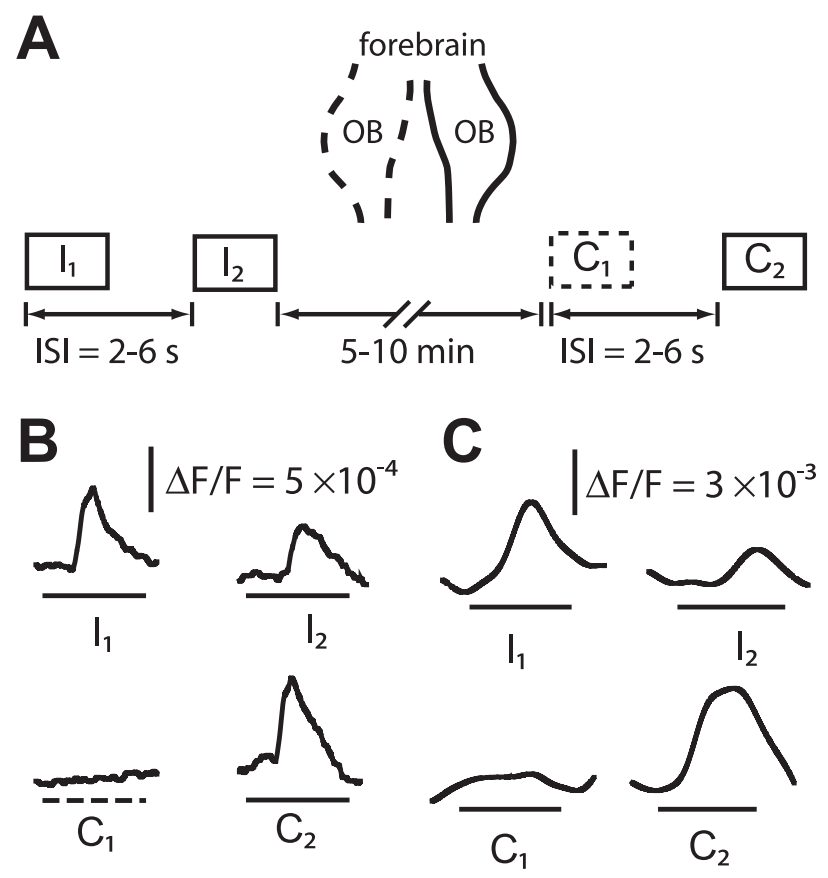

D

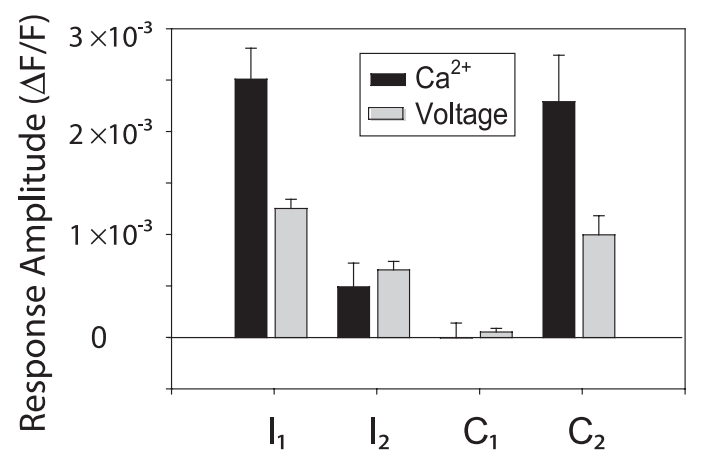

FIG. 1. Ipsilateral and contralateral paired-pulse stimulation of the olfactory system. (A) Schematic of stimulation scheme. Only one of the paired OBs was imaged (solid outline). In ipsilateral paired-pulse stimulation (IPPS), a conditioning pulse $\left(\mathrm{I}_{1}\right)$ of odorant was selectively delivered to the naris ipsilateral to the imaged OB. Following an ISI of $2-6 \mathrm{~s}$, a test pulse $\left(\mathrm{I}_{2}\right)$ was delivered to the same naris. In contralateral paired-pulse stimulation (CPPS), a conditioning pulse $\left(\mathrm{C}_{1}\right)$ of odorant was first delivered on the side of the unimaged $\mathrm{OB}$. The test pulse $\left(\mathrm{C}_{2}\right)$ was delivered on the side of the imaged $\mathrm{OB}$. All paired-pulse trials were separated by a period of $5-10 \mathrm{~min}$ to minimize interaction between trials. (B) Voltage-sensitive dye measurement of localized OB response, averaged over five adjacent pixels. During IPPS, responses to both $I_{1}$ and $I_{2}$ were recorded. During CPPS there was no response to $C_{1}$, consistent with selective stimulation of the unimaged $\mathrm{OB}$, and a response to $\mathrm{C}_{2}$ was recorded. Horizontal bars indicate $1 \mathrm{~s}$ pulses of odorant. The odorant was cineole and the ISI was $5 \mathrm{~s}$. (C) $\mathrm{Ca}^{2+}$-sensitive dye measurement of localized $\mathrm{OB}$ response, averaged over five adjacent pixels. As observed in the voltagesensitive dye measurement there was no response to $C_{1}$, indicating that there was no ORN input to the imaged $\mathrm{OB}$ during the first pulse of contralateral stimulation. The odorant was cineole and the ISI was $4 \mathrm{~s}$. (D) $\mathrm{Ca}^{2+}-$ sensitive dye measurement of ORN terminal responses (solid) and voltagesensitive dye measurement of OB responses (shaded) to IPPS and CPPS. The response to $I_{2}$ was smaller than the response to $I_{1}$. No response was seen to $C_{1}$, and the responses to $I_{1}$ and $C_{2}$ were not different (see Results). Thus, while paired-pulse inhibition of ORN activity may play a role in modulation of $\mathrm{I}_{2}$ responses relative to $I_{1}$, the response to $C_{2}$ is not conditioned by stimulation of ORNs or activation of the $\mathrm{OB}$ on $\mathrm{C}_{1}$. Odorants were cineole and isoamyl acetate, and the ISI was $4 \mathrm{~s}$. Calcium responses were recorded from $n=3$ animals, while voltage-sensitive dye responses were recorded from $n=12$ animals. recordings from a single animal. Response amplitude was calculated as the difference between baseline (average response over $400 \mathrm{~ms}$ preceding the odorant pulse) and peak (average response over $200 \mathrm{~ms}$ surrounding a visible peak) responses. In control trials where no responses were visible, the peak response was defined by averaging over a $200 \mathrm{~ms}$ window centred $500 \mathrm{~ms}$ after the odorant pulse.

\section{Voltage-sensitive dye responses}

For illustrative traces presented in Figs 1,2 and $5, \Delta \mathrm{F} / \mathrm{F}$ was calculated by division by resting fluorescence and responses were digitally filtered at $1-10 \mathrm{~Hz}$. The results depicted are averages of 5-10 adjacent pixels, and the same set of pixels was used for all traces illustrated within a single figure.

For quantitative analyses of voltage-sensitive dye signals in Figs 3-5, the voltage output of the photodiode array was internally normalized (see Activation, below). The signal from each pixel was considered individually, without spatial averaging, and filtered with low- and high-pass Gaussian filters at $1-10 \mathrm{~Hz}$. The output of the photodiode array was spatially masked for each trial so that only pixels overlying the $\mathrm{OB}$ were included in the analysis.

\section{Activation}

The response to odorant stimulation in the turtle includes both a longlasting depolarization (DC signal) and oscillatory components (Lam et al., 2000; Lam et al., 2003). Here, we analysed the dynamics of the ascending phase of the long-lasting depolarization as a measure of $\mathrm{OB}$ activation. We defined activation as the root-mean-squared deviation of the ascending phase of the depolarization:

$$
A_{i}^{p}=\sqrt{\frac{1}{N-1} \sum_{t=t_{\text {base }}}^{t_{\text {peak }}}\left(x^{p}(t)-\bar{x}^{p}\right)^{2}}
$$

Here, the superscript index $p$ denotes that the calculation is being performed for a single pixel, and the subscript $i$ indicates the odorant pulse. The indices $t_{\text {base }}$ and $t_{\text {peak }}$ refer to the base and peak of the ascending phase of the depolarization, which are determined by visual inspection. The values of the time series at a single pixel within this region are denoted by $x^{p}(t)$, while $\bar{x}^{p}$ is their temporal average and $N$ is the total number of sampled points. In recordings with little baseline noise, this measure performed similarly to the amplitude of the depolarization ( $r=0.992,260$ pixels).

\section{Similarity of response patterns}

In order to evaluate the reproducibility of $\mathrm{OB}$ activity patterns produced in response to odorant stimulation we calculated the highdimensional angular distance between pairs of imaged responses. For two responses, we calculated the amplitude of the DC response in voltage-sensitive dye recordings, extracted those pixels overlying the $\mathrm{OB}$, and created two response vectors, $\mathrm{R}_{1}$ and $\mathrm{R}_{2}$. The corresponding elements of these vectors describe activation at the same physical location in the photodiode array. We then calculate the angular separation $(\theta)$ of these two vectors, which measures the similarity of spatial patterning in the two responses:

$$
\theta=\cos ^{-1}\left(\frac{\left\langle R_{1}, R_{2}\right\rangle}{\left\|R_{1}\right\| \times\left\|R_{2}\right\|}\right)
$$

Amplitude was used in place of our activation measure in order to accurately capture changes in the voltage-sensitive dye signal during noisy recordings in the absence of odor stimulation, which may show responses of both positive and negative amplitude. 


\section{Changes in activation}

For the purposes of measuring mean inhibition or potentiation of the $\mathrm{OB}$ response, we defined the change in activation

$$
\Delta A_{i, j}=\left\langle\frac{A_{j}^{p *}-A_{i}^{p *}}{A_{j}^{p *}+A_{i}^{p *}}\right\rangle_{p *}
$$

where the subscripts $i$ and $j$ refer to the activation in response to two different odorant pulses. In order to compensate for small

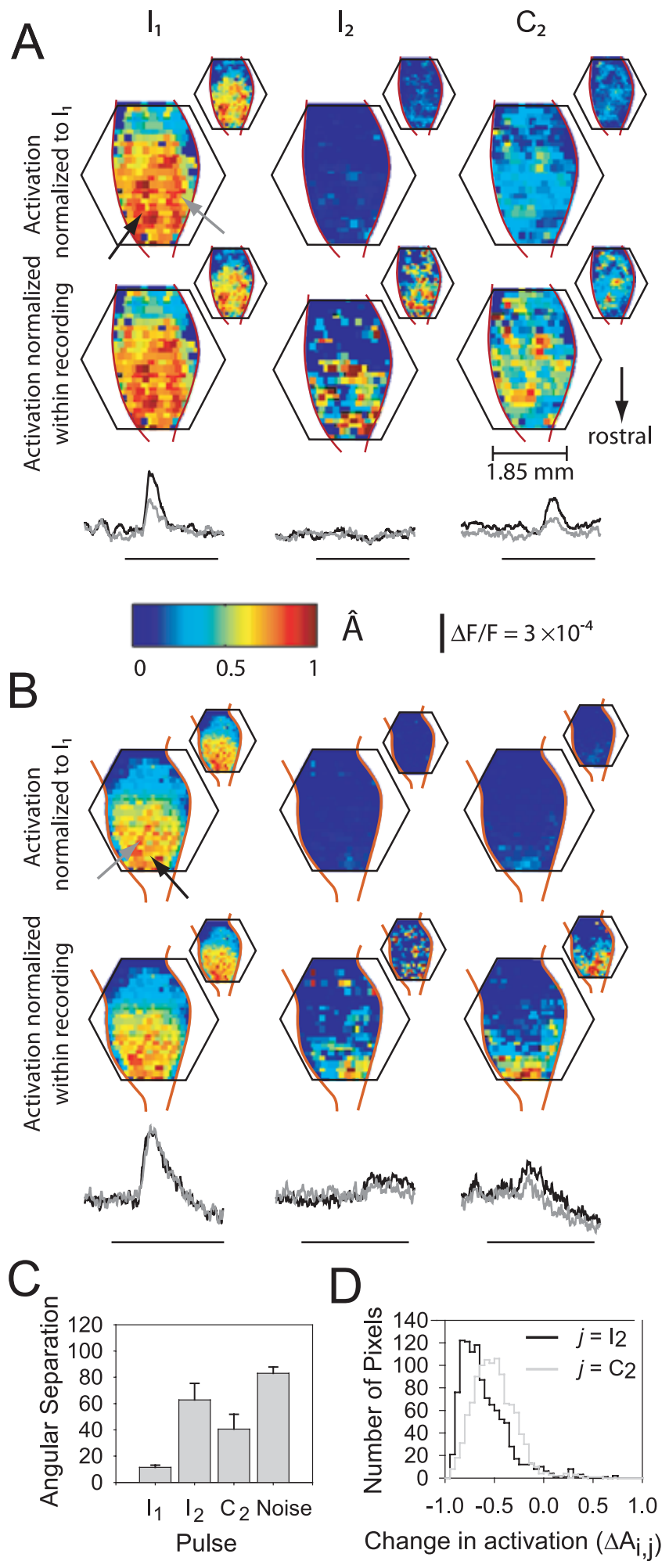

changes in image registration between pulses, pixels were compared on the basis of activation rank rather than strictly on their position in the photodiode array. The rank of each pixel's activation in response to a particular odorant pulse was calculated, and pixels with the same rank (denoted $p^{*}$ ) were compared across odorant pulses. Spatial maps of rank values for each odorant pulse were visually compared to ensure that this procedure did not result in the pairing of pixels from distant regions of the OB. Similar results were obtained by first averaging the activation values at all locations overlying the $\mathrm{OB}$ and then computing the change in average values. The measure $\Delta A_{\mathrm{i}, \mathrm{j}}$ is the spatial average of these comparisons over all pairs $p^{*}$, and takes values between -1 and 1 . This measure avoids infinite responses which would result from taking simple ratios in the case of very small activations such as those observed in Fig. 5.

\section{Spatial normalization of activation}

In order to highlight spatial differences in activation in response to a single odorant pulse, we define the normalized activation

$$
\hat{A}_{i}^{p}=\frac{A_{i}^{p}}{\max _{p}\left(A_{i}^{p}\right)}
$$

Normalizing the activation at each pixel by the maximum observed activation to a given odorant pulse results in values for $\hat{A}_{i}^{p}$ between 0 and 1 .

\section{Changes in contrast}

Changes in the spatial contrast of activation were observed by comparing cumulative distribution functions $\left(C_{i}(\hat{A})\right)$ of normalized activation values at each pixel $\left(A_{i}^{p}\right)$ value among the responses to two different odorant pulses. A shift of the cumulative distribution function to the left corresponds to an increase in contrast. Changes in contrast were quantified over multiple such comparisons by measuring the area between the cumulative distribution function curves corresponding to the first and second odor pulses:

FIG. 2. Examples of the spatial distribution of activation in the OB. Images represent pixel-wise displays of normalized activation in response to individual pulses of odorant stimulation. The spatial extent of the photodiode array (black hexagon) and dorsal surface of the OB (red outline) are shown schematically. (A, upper) Activation at each pixel is normalized to the maximum activation observed in response to $I_{1}$, showing the inhibition of $I_{2}$ and $C_{2}$ responses relative to the $I_{1}$ response. (A, middle row) Activation at each pixel is normalized to the maximum activation observed within the same image, highlighting the spatial distribution of relative activation within each image. Areas of maximal activation on $\mathrm{I}_{2}$ and $\mathrm{C}_{2}$ are spatially constrained relative to $\mathrm{I}_{1}$, indicating increased contrast. The odorant was cineole and the ISI was $2 \mathrm{~s}$. (Insets) To demonstrate the repeatability of the spatial pattern of activation, an additional set of odorant stimulation responses from the same animal are shown. The odorant was cineole and the ISI was $2 \mathrm{~s}$. (A, Lower row) Time courses of the local voltage-sensitive dye signal taken from two areas of the $\mathrm{OB}$ (arrows). The $1 \mathrm{~s}$ odorant stimuli are indicated by the horizontal lines. (B) Spatial activation maps and time courses in a different animal than those shown in A. The odorant was cineole and ISIs were $2.5 \mathrm{~s}$ (upper and middle rows) and $2 \mathrm{~s}$ (insets). (C) Angular separation of spatial patterns for IPPS and CPPS trials with ISIs of 2 and $2.5 \mathrm{~s}$. The responses to $I_{1}$ and $C_{2}$ were significantly more self-similar within individual animals than the noisy baseline signal, indicating the repeatability of the spatial pattern of activation. The odorants were cineole and isoamyl acetate; $n=4$ animals. (D) Distribution of values for the change of activation $\left(\Delta A_{\mathrm{i}, \mathrm{j}}\right)$ at each pixel in the activation maps shown in $\mathrm{A}$ and $\mathrm{B}$, above. The response to IPPS (black) displays greater inhibition than the response to CPPS (grey), but both distributions are broad, suggesting that inhibition of the OB response is not spatially uniform. 


$$
\Delta C_{i, j}=\int_{0}^{1}\left[C_{j}(\hat{A})-C_{i}(\hat{A})\right] d \hat{A}
$$

Thus, negative values of this measure correspond to a loss of contrast while positive values correspond to contrast enhancement.

Another way of visualizing changes in contrast is to plot changes in $\hat{A}_{i}^{p}$ as a function of $\hat{A}_{i}^{p}$ values (see Fig. 3). Rank-wise comparison of pixels is mathematically equivalent to direct subtraction of cumulative
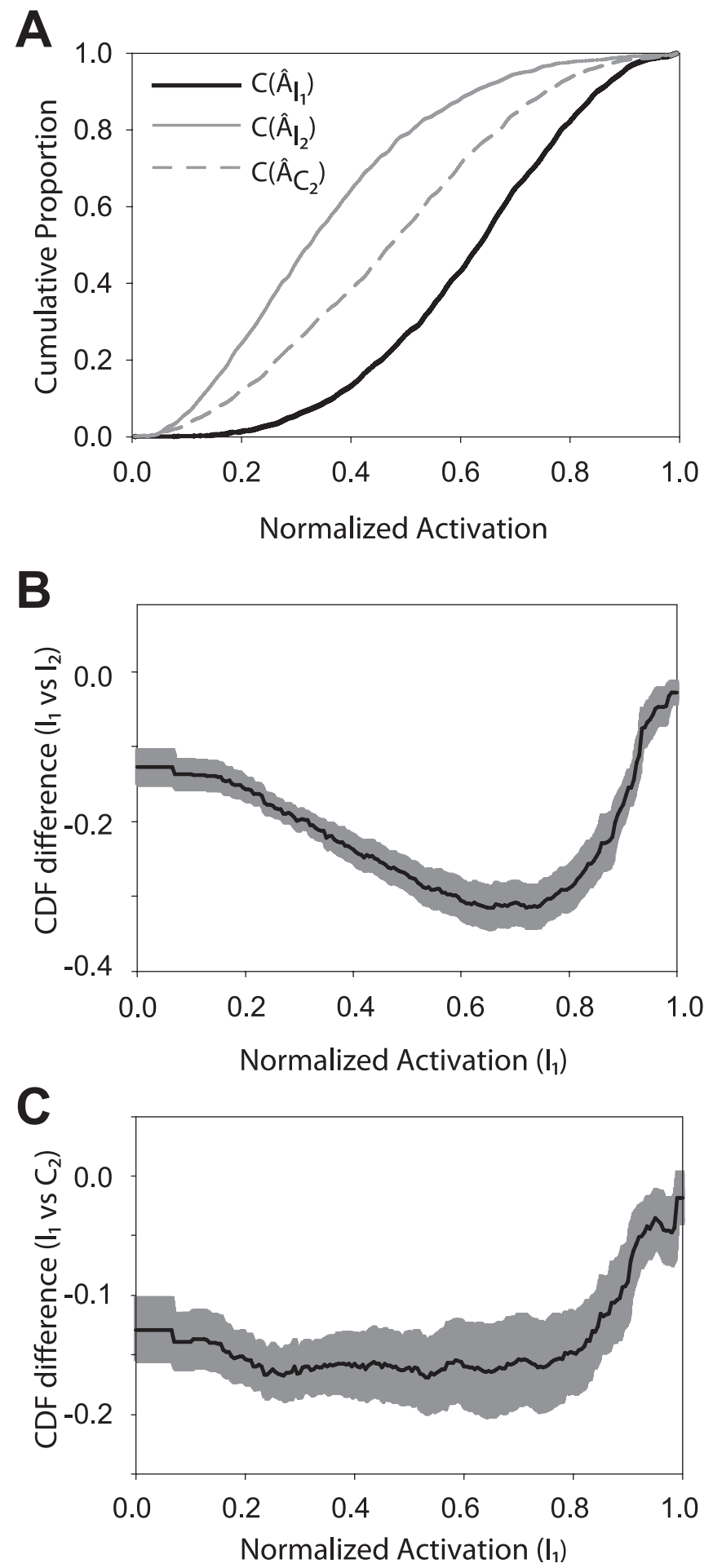

distribution functions. The responses to two odorant pulses were compared by pairing pixels rank-wise (see above), and plotting $\hat{A}_{j}^{p *}-\hat{A}_{i}^{p *}$ against $\hat{A}_{i}^{p *}$. For the purpose of this subtraction, the point $\left(\hat{A}_{j}^{p *}, \hat{A}_{i}^{p *}\right)=(1.0,1.0)$ is discarded.

\section{Statistical tests}

In order to account for intra-animal correlation in our results, comparisons of $\mathrm{Ca}^{2+}$ signal amplitude and voltage-sensitive dye measurements of inhibition and contrast were carried out using a mixed-effects linear model. This test was structured to evaluate random effects of individual animals and fixed effects of interstimulus interval (ISI), odorant, and ipsilateral vs. contralateral stimulation. We report the results of this test with an $F$ statistic with degrees of freedom and $P$-value, and $n$-values are given for the number of animals. Post hoc comparisons were carried out using two-tailed Student's $t$-tests unless noted otherwise. Statistical tests were performed using built-in functions in SPSS (SPSS Inc., Chicago, IL, USA). In all figures, error bars indicate 1 SEM.

\section{Results}

Paired-pulse stimulation delivered to a single OB results in complex responses throughout the olfactory system, from ORN to olfactory cortex (Getchell, 1986; Schafer et al., 2005; Zochowski \& Cohen, 2005; Kadohisa \& Wilson, 2006). We isolated the effects of pairedpulse stimulation on the centrifugal projections of retrobulbar structures by delivering a conditioning odorant stimulus to the contralateral, unimaged, OB.

We made voltage-sensitive dye recordings to monitor the spatial distribution of $\mathrm{OB}$ activation in response to paired pulses of odorant stimulation. The changes in dye fluorescence, imaged by a 464element photodiode array, provided a fast measure of the average membrane potential changes in the populations underlying each pixel (Ross et al., 1977; Grinvald et al., 1988; Zochowski et al., 2000). A single $\mathrm{OB}$ was imaged. In ipsilateral paired-pulse stimulation (IPPS), a $1 \mathrm{~s}$ pulse of odorant $\left(\mathrm{I}_{1}\right)$ was selectively delivered to the naris ipsilateral to the imaged $\mathrm{OB}$ (Fig. 1A and B). Following an ISI of 2-6 s, a test pulse $\left(\mathrm{I}_{2}\right)$ was delivered to the same naris.

In contralateral paired-pulse stimulation (CPPS), a conditioning pulse $\left(\mathrm{C}_{1}\right)$ of odorant was first delivered on the side of the unimaged OB. The test pulse $\left(\mathrm{C}_{2}\right)$ was delivered on the side of the imaged OB. Thus, we recorded three responses of the same OB to stimulation: $\mathrm{I}_{1}$, $I_{2}$ and $C_{2}$ (Fig. 1B). The responses to $I_{1}$ were taken as a common baseline for comparison with both the ipsilaterally conditioned $\mathrm{I}_{2}$ and contralaterally conditioned $\mathrm{C}_{2}$ responses in subsequent analyses.

FIG. 3. Paired-pulse stimulation increases spatial contrast in OB activation. (A) Cumulative density function constructed from pooled values of $\hat{A}_{i}^{p}$ across multiple trials of IPPS and CPPS with ISI $=2 \mathrm{~s}$. The cumulative density function of $\mathrm{I}_{2}$ and $\mathrm{C}_{2}$ responses are shifted to the left of the $\mathrm{I}_{1}$ response, indicating increased contrast (see Results). (B) Transfer function representation of the relationship between $I_{1}$ and $I_{2}$ above. For IPPS trials, pixels in the $I_{1}$ and $\mathrm{I}_{2}$ images are paired rank-wise by their $A_{\text {norm }}$ values (see Materials and methods). The difference $\hat{A}_{\mathrm{I}_{2}}^{p *}-\hat{A}_{\mathrm{I}_{1}}^{p *}$ is plotted against the value of $\hat{A}_{\mathrm{I}_{1}}^{p *}$ on a pixel-by-pixel basis. The function shows that high and low values of activation are preserved relative to intermediate values, a hallmark of contrast enhancement. Mean and SEM are represented by the black line and grey shaded area. (C) Transfer function representation of the relationship between $I_{1}$ and $C_{2}$ above. The function shows that high values of activation are preserved relative to intermediate values, a hallmark of contrast enhancement. Mean and SEM are represented by the black line and grey shaded area. 
All paired-pulse trials were separated by a period of 5-10 min to minimize habituation between trials. IPPS and CPPS trials utilizing the same ISI were performed consecutively in order to minimize variability due to bleaching of the voltage-sensitive dye and phototoxicity (Zochowski et al., 2000). Although paired trials were consecutive, IPPS trials did not necessarily precede CPPS trials.
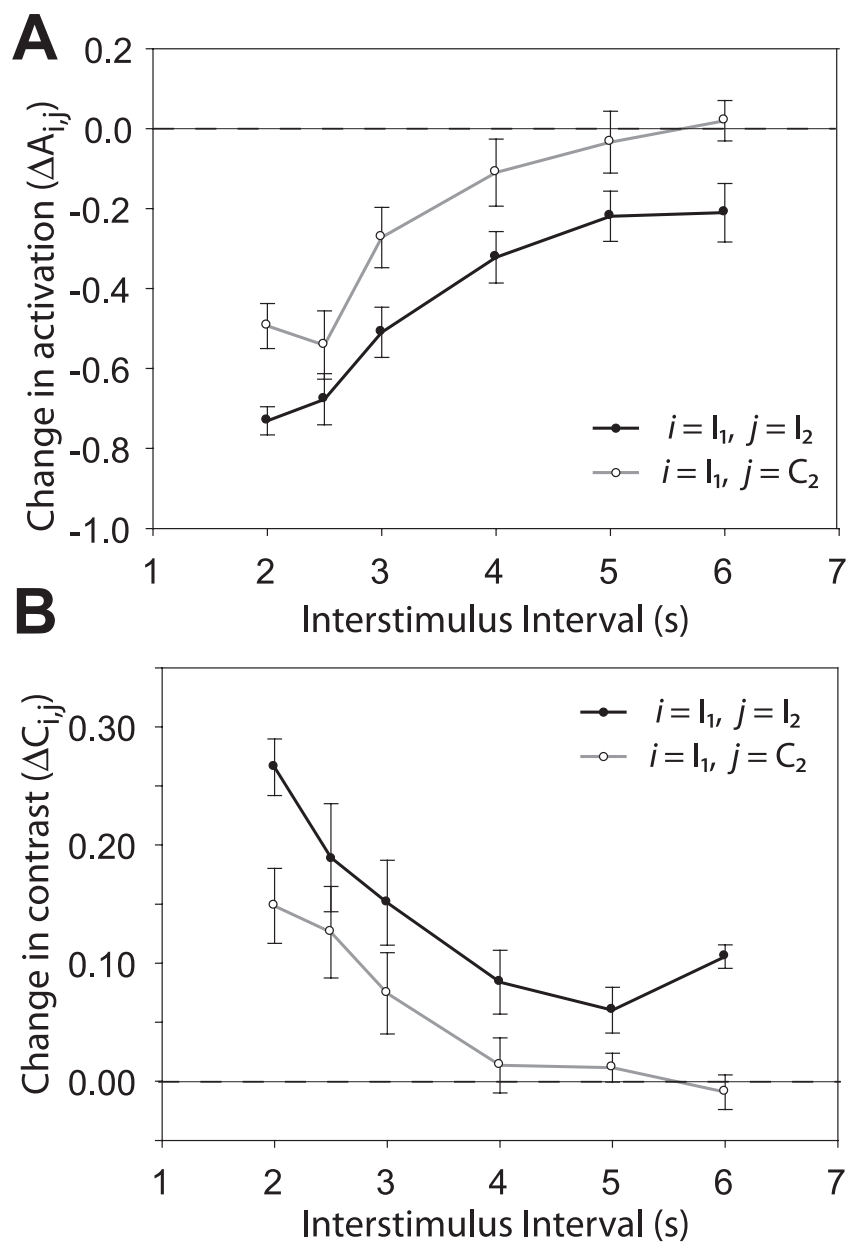

FIG. 4. Inhibition and contrast enhancement varied with ISI. (A) Mean change in activation over the $\mathrm{OB}$, comparing $\mathrm{I}_{2}$ and $\mathrm{C}_{2}$ responses to the common baseline of $\mathrm{I}_{1}$. Both IPPS (black) and CPPS (grey) resulted in significant inhibition at short ISIs, which decayed for longer ISIs (see Results). (B) Change in contrast, comparing $\mathrm{I}_{2}$ and $\mathrm{C}_{2}$ responses to the common baseline of $\mathrm{I}_{1}$ over a range of ISIs. Both IPPS (black) and CPPS (grey) resulted in significant contrast enhancement at short ISIs (see Results).

FIG. 5. Pairing different odorants enhanced inhibition. (A) Spatial activation maps and local (black arrow) time course signals in the same animal in response to different odorant stimuli. The normalized activation $(\hat{\mathrm{A}})$ is computed relative to the maximal response to $\mathrm{I}_{1}$ stimulation in each set. The ISI was $4 \mathrm{~s}$, an interval at which little inhibition of $I_{2}$ and $C_{2}$ relative to the $I_{1}$ response is expected (see Fig. 4). (Upper and middle) After IPPS and CPPS with a $1 \mathrm{~s}$ (horizontal line) presentation of a single odorant (upper, cineole; middle, isoamyl acetate), responses to $\mathrm{I}_{2}$ and $\mathrm{C}_{2}$ were not inhibited relative to $I_{1}$. (Lower) In response to IPPS and CPPS with presentation of isoamyl acetate on the first pulse and cineole on the second, the $\mathrm{I}_{2}$ and $\mathrm{C}_{2}$ responses were strongly inhibited relative to $\mathrm{I}_{1}$. The animal and ISI were the same as above. (B) For both IPPS and CPPS, mean inhibition increased when the odorant presented on $\mathrm{I}_{2}$ or $\mathrm{C}_{2}$ was different that the odorant presented on $\mathrm{I}_{1}$ and $\mathrm{C}_{1}$. ISI is $4 \mathrm{~s}$ and $n=5$ animals.
After the preparation of each turtle, the response of each OB to a $1 \mathrm{~s}$ pulse of $7 \%$ cineole and a $1 \mathrm{~s}$ pulse of $7 \%$ isoamyl acetate was tested to ensure that both OBs responded to odorant stimulation. A single OB was then selected for imaging for the duration of the experiment. In most animals, IPPS and CPPS responses to both cineole and isoamyl acetate were recorded. The responses to the two odorants were pooled for analysis.
A

A

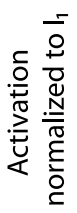
$\mathrm{l}_{1}$

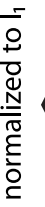
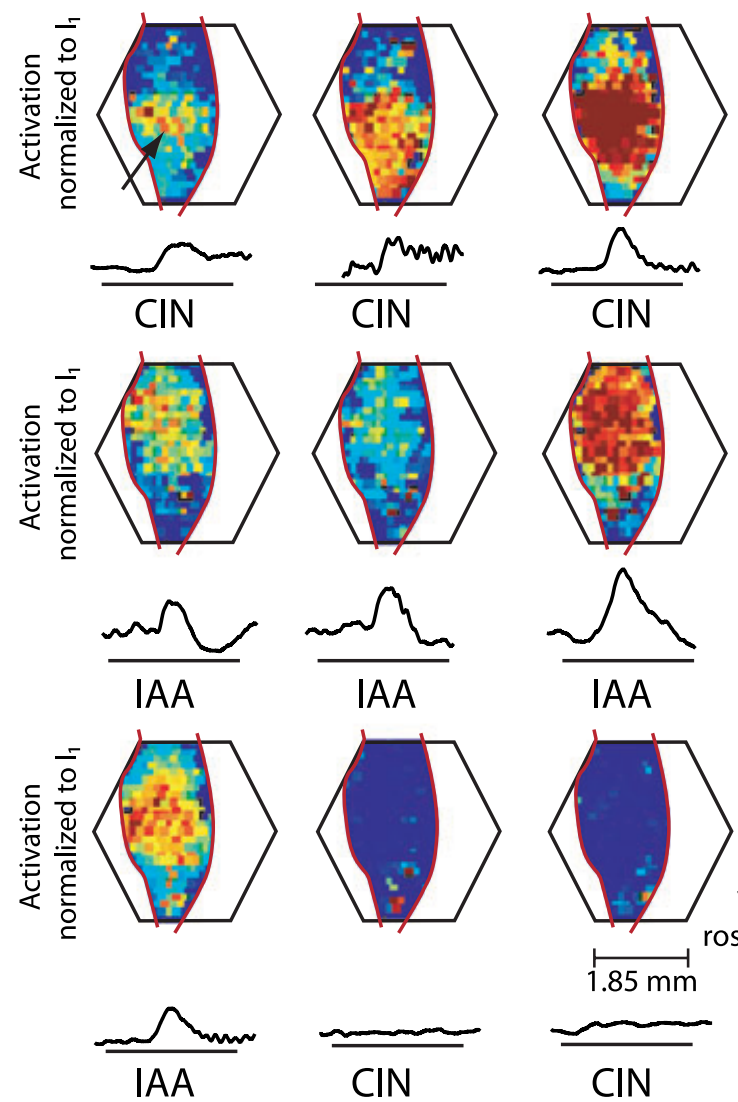

$$
\mathrm{l} \Delta \mathrm{F} / \mathrm{F}=8 \times 10^{-4}
$$

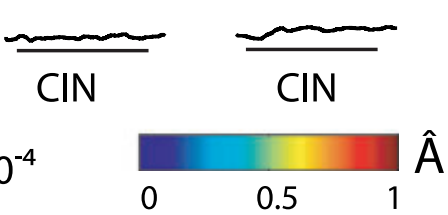

B

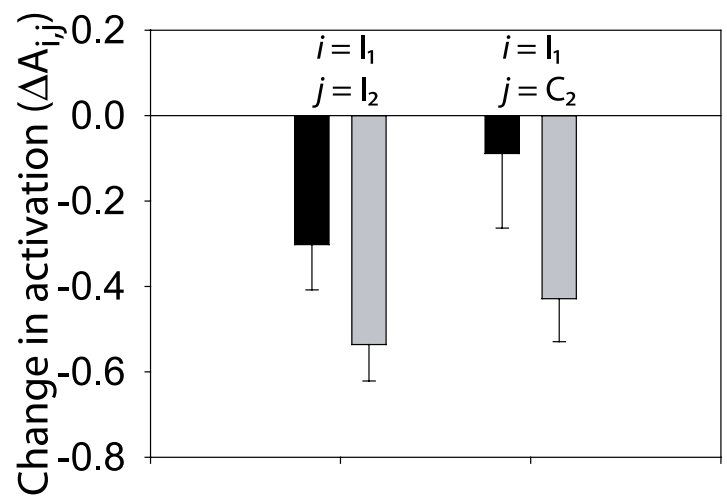

Same odorant on 1st, 2nd pulses Different odorant on 1st, 2nd pulses 
To control for the possibility that odorant delivered to the naris contralateral to the imaged $\mathrm{OB}$ also leaked into the ipsilateral naris, the imaged response to $\mathrm{C}_{1}$ stimulation was evaluated for each CPPS trial. Trials were only included in the analysis if there was no response to the $\mathrm{C}_{1}$ stimulus, indicating that the imaged $\mathrm{OB}$ had not been stimulated (Fig. 1B). To demonstrate quantitatively that the voltagesensitive dye signal did not show any response to the $C_{1}$ stimulus, we compared the responses to $I_{1}, I_{2}$ and $C_{2}$ pulses with the activation observed $500 \mathrm{~ms}$ after the onset of the $\mathrm{C}_{1}$ stimulus to the contralateral bulb. Responses to IPPS and CPPS recorded from 12 animals using both cineole and isoamyl acetate stimuli and an ISI of $4 \mathrm{~s}$ are shown (Fig. 1D, shaded bars). Responses to the four pulses were different ( $\left.n=12, F_{3,56}=21.05, P<0.001\right)$. The average response amplitude to $\mathrm{C}_{1}$ stimulation was smaller than the response to all three other pulses $(P<0.005)$. Furthermore, the amplitude of the $\mathrm{C}_{1}$ response was not significantly different from $0(P>0.1)$. Given the variance of the responses, this analysis had the power to detect a difference from 0 of amplitude $\Delta \mathrm{F} / \mathrm{F}<1 \times 10^{-4}(\alpha=0.05, \beta=0.05)$. Some trials did exhibit artifactual responses to $C_{1}$ stimulation due solely to movement of the turtle during imaging. The $\mathrm{I}_{1}, \mathrm{I}_{2}$ and $\mathrm{C}_{2}$ responses from these trials were included in analysis if the artifact was visible both over the $\mathrm{OB}$ and additionally over adjacent imaged structures, such as dura mater and bone, indicating that there was no stimulation of the ipsilateral $\mathrm{OB}$ during the $\mathrm{C}_{1}$ stimulus. Trials with movement artifacts in $\mathrm{I}_{1}, \mathrm{I}_{2}$, or $\mathrm{C}_{2}$ responses were excluded.

To further show that our CPPS procedure did not result in activation of the imaged $\mathrm{OB}$ during $\mathrm{C}_{1}$, we imaged the presynaptic activation of ORN input to the OB using a calcium-sensitive dye (Wachowiak \& Cohen, 1999). We found no response of the imaged OB to stimulation of the contralateral OB (Fig. 1C). Responses to IPPS and CPPS over multiple trials with ISI $=4 \mathrm{~s}$ in three animals are shown (Fig. 1D, solid bars). Responses to cineole and isoamyl acetate are pooled. The responses to the four stimulus pulses were different $(n=3$, $\left.F_{3,61}=16.67, P<0.001\right)$ while the identity of the stimulus odorant showed no effect $\left(F_{1,61}=0.349, P>0.5\right)$. The responses to $\mathrm{I}_{1}$ and $\mathrm{C}_{2}$ were not different $(P>0.5)$, while the response to $\mathrm{C}_{1}$ was much less $(P<0.001)$. The amplitude of the $\mathrm{C}_{1}$ response was not significantly different from $0(P>0.5)$. Given the variance of the responses, this analysis had the power to detect a difference from 0 of amplitude $\Delta \mathrm{F} / \mathrm{F}<5 \times 10^{-4}(\alpha=0.05, \beta=0.05)$. These results suggest that there is no conditioning of the ORN input to the imaged OB by the contralateral stimulus $\mathrm{C}_{1}$.

\section{Changes in spatial patterning after paired-pulse stimulation}

When we compared the conditioned voltage-sensitive dye responses of the imaged $\mathrm{OB}$ to stimuli $\mathrm{I}_{2}$ and $\mathrm{C}_{2}$ with the baseline response $\mathrm{I}_{1}$, we observed striking changes in the spatial pattern of $\mathrm{OB}$ activation (Fig. 2A and B). To ensure that these changes represent reproducible modulation of the $\mathrm{OB}$ response and not artifactual fluctuations in the recorded signals, we show the response to a replicated set of IPPS and CPPS trials in the same animals with the same ISI (Fig. 2A and B, insets). While these maps appear similar, we further quantified the reproducibility of $\mathrm{OB}$ activation patterns by calculating the angular separation of OB responses from pairs of IPPS and CPPS trials with the same odorant and ISI of 2 or $2.5 \mathrm{~s}$ repeated in the same animal. We calculated the angular separation for responses to $I_{1}, I_{2}$ and $C_{2}$. For comparison, we also calculated the angular separation of maps generated from baseline fluctuations during a period preceding odor stimulation (Fig. 2C). These comparisons were carried out in four animals. We found that the degree of reproducibility during these four epochs was different $\left(F_{3,16}=12.2, P<0.001\right)$ with responses to $\mathrm{I}_{1}$ and $\mathrm{C}_{2}$ being more reproducible (less angular separation among replicates) than the response to noise $(P<0.001, P<0.05$, respectively). The response to $\mathrm{I}_{2}$ was not significantly more reproducible than noise. We attribute this disparity to a floor effect on our measurement: the highly inhibited responses at very short ISIs are of an amplitude roughly similar to baseline fluctuations in some cases.

Two classes of changes were evident when we compared the baseline $\left(\mathrm{I}_{1}\right)$ and conditioned $\left(\mathrm{I}_{2}\right.$ and $\left.\mathrm{C}_{2}\right)$ responses. First, the average level of activation imaged across the $\mathrm{OB}$ was greatly decreased, suggesting a broad suppression of the $\mathrm{OB}$ response (Fig. 2A and B, upper). We will refer to this effect here as 'inhibition', although it may arise from a number of neurophysiological processes (see Discussion). Second, the spatial extent of highly activated regions (red pixels) was more limited after either a contralateral or ipsilateral conditioning stimulus (Fig. 2A and B, middle). This shift to a smaller area of relative high normalized activation indicates an enhancement of the spatial contrast in the imaged activity. In time-series views of the voltage-sensitive dye signal from different locations of the dorsal OB (Fig. 2A and 2B, lower), we observed both different response amplitudes to $I_{1}$ and differing levels of inhibition in response to subsequent pulses, at different locations on the OB. We calculated the change in activation $\left(\Delta A_{i, j}^{p}\right)$ for nonnormalized activation values at each pixel of the photodiode array for the four sets of example maps shown. We found that the value of this change in activation fell in a very broad distribution, indicating that the degree of inhibition observed was highly nonuniform across the surface of the OB (Fig. 2D). While uniform inhibition across the surface of the OB would not lead to changes in activation map contrast, such spatially differentiated inhibition changes the spatial pattern of activation.

In order to quantify the change in spatial contrast following a conditioning odor stimulus, we constructed cumulative density functions $(C)$ from the normalized activation values at each pixel $\left(\hat{A}_{i}^{p}\right)$ during the responses to stimuli $\mathrm{I}_{1}, \mathrm{I}_{2}$ and $\mathrm{C}_{2}$. An example of such a set of cumulative density functions, calculated from the pooled $\hat{A}_{i}^{p}$ values of all trials with ISI $=2 \mathrm{~s}$, is shown in Fig. 3A. The locations of the three distributions are different, with distribution of $\mathrm{I}_{2}$ responses shifted most to the left, followed by $\mathrm{C}_{2}$ and $\mathrm{I}_{1}$ (Kruskal-Wallis test, $P<0.001)$. A shift to the left of the cumulative distribution indicates an increase in contrast, as pixels of intermediate normalized activation shift to lower values. In subsequent analyses, we thus quantified a change in contrast $\left(\Delta C_{\mathrm{i}, \mathrm{j}}\right)$ as the area between the cumulative distribution functions $C_{\mathrm{i}}$ and $C_{\mathrm{j}}$ for two different stimulus pulses.

To further visualize the contrast-enhancing effects of a conditioning odor stimulus, we calculated the difference between the cumulative distribution functions and plotted them against the normalized activation observed on $I_{1}$ to form a transfer function representing the changes in the distribution of activation after IPPS or CPPS. We found that for IPPS the transfer function preserved high activation at the expense of intermediate and low activation (Fig. 3B). This pattern forms a 'half-hat' function, a hallmark of contrast enhancement (Urban, 2002; Cleland \& Sethupathy, 2006). The same pattern, although with a lesser degree of inhibition, was observed following CPPS (Fig. 3C).

Both the broad inhibitory and contrast-enhancing effects of IPPS and CPPS were long-lived. Varying the ISI from 2 to $6 \mathrm{~s}$ revealed a steady decrease in inhibition and contrast enhancement (Fig. 4). Contralateral stimulation evoked less inhibition than did IPPS (Fig. $4 \mathrm{~A} ; n=18$ animals, $F_{1,154}=23.5, P<0.001$ ). The degree of inhibition changed with ISI $\left(F_{5,154}=15.4, P<0.001\right)$. After IPPS, the change in activation $\left(\Delta A_{\mathrm{I}_{1}}, \mathrm{I}_{2}\right)$ was significantly $<0$ for ISI values from 2 to $5 \mathrm{~s}$, while the inhibition observed following CPPS $\left(\Delta A_{\mathrm{I}_{1}}, \mathrm{C}_{2}\right)$ 
was nonzero for ISI values from 2 to $3 \mathrm{~s}$ (one-sample $t$-tests, $P<0.05)$.

Contrast enhancement was also greater following IPPS than CPPS (Fig. 4B; $F_{1,154}=25.1, P<0.001$ ). Contrast enhancement decreased with increasing ISI $\left(F_{5,154}=11.9, P<0.001\right)$. The change in contrast following IPPS $\left(\Delta C_{\mathrm{I}_{1}}, \mathrm{I}_{2}\right)$ was significantly different from zero for all ISI values measured, while the change following CPPS $\left(\Delta C_{\mathrm{I}_{1}}, \mathrm{C}_{2}\right)$ was nonzero only for ISI $=2$ and $2.5 \mathrm{~s}$ (independent sample $t$-test, $P<0.05)$.

\section{Inhibition and contrast were enhanced by conditioning with different odorants}

We hypothesized that the contrast enhancement observed in the spatial patterning of OB activation reflects a sharpening of odorant representations within the OB through inhibitory mechanisms (Yokoi et al., 1995; Meister \& Bonhoeffer, 2001). If this is the case, then administering different odorants as the conditioning $\left(\mathrm{I}_{1}\right.$ and $\left.\mathrm{C}_{1}\right)$ and test $\left(\mathrm{I}_{2}\right.$ and $\left.\mathrm{C}_{2}\right)$ stimuli should result in greater mean inhibition and contrast enhancement than if a single odorant were used. This would occur if the conditioning stimulation evoked inhibition of all $\mathrm{OB}$ regions not involved in the coding of the conditioning odorant. Upon subsequent stimulation with a second, different, odorant then only the OB regions that coded for both odorants would be activated.

To test this hypothesis, we measured $\mathrm{OB}$ responses in five turtles using a different odorant stimulus during the $\mathrm{I}_{1}$ and $\mathrm{C}_{1}$ pulses than during the $\mathrm{I}_{2}$ and $\mathrm{C}_{2}$ pulses. At the outset of the experiment, IPPS and CPPS responses to both cineole and isoamyl acetate were confirmed (for example, Fig. 5A). We then stimulated with either cineole or isoamyl acetate during $I_{1}$ and $C_{1}$, and the other odorant during $I_{2}$ and $\mathrm{C}_{2}$. We also performed the reciprocal experiment, reversing the order of odorant presentation. The ISI used was $4 \mathrm{~s}$, which in single-odorant experiments evoked only a weak inhibition and contrast enhancement (Fig. 4).

Stimulating with different odorants as the conditioning and conditioned stimuli resulted in a dramatic increase in inhibition. With an ISI of $4 \mathrm{~s}$, there was robust activation and very little inhibition evident after IPPS and CPPS in response to both cineole and isoamyl acetate (Fig. 5A, upper, middle). This was evident both in time series from an individual region and in activation maps showing widespread high levels of activation. In the following trials in the same animal, however, stimulating with a different odorant in the second pulse led to a dramatic reduction in activation (Fig. 5A, lower). The suppression of the $\mathrm{I}_{2}$ and $\mathrm{C}_{2}$ responses was so great that there was little spatial patterning evident and thus we could not measure changes in contrast. In an analysis of five animals, however, we observed greater inhibition when different odorant stimuli were used than when the same odorant was used (Fig. 5B, matched-pairs comparison within animal; onetailed $t$-test, $P<0.05)$.

\section{Discussion}

We have found that both ipsilateral and contralateral conditioning odorant stimuli elicit a profound contrast-enhancing inhibition of the $\mathrm{OB}$ response to a test stimulus. Furthermore, we have found that inhibition is increased if the conditioning and test stimuli utilize different odorants, suggesting a possible functional role for centrally mediated inhibition in odor discrimination.

The inhibition and contrast enhancement observed after ipsilateral conditioning could be due to a number of processes, including adaptation of ORN fibers and activation or habituation of intrinsic OB networks persisting from the conditioning stimulus to the test stimulus response. Indeed, we found that the sum of these processes can often reduce $\mathrm{OB}$ activation below the level of detection after ipsilateral conditioning, making measurement of spatial patterning difficult. Here, our goal was to isolate the action of retrobulbar feedback from these myriad mechanisms. While the response to IPPS provides an interesting point of comparison, in this study we emphasize the finding that retrobulbar feedback, as measured by the response to CPPS, resulted in contrast-enhancing inhibition.

The reciprocal inhibitory influences of the paired OBs have long been recognized (Walsh, 1959; Kerr, 1960; Leveteau \& MacLeod, 1969; Leveteau et al., 1971). This inhibition has been attributed to centrifugal fibers of the anterior olfactory nucleus exciting the inhibitory granule cells of the OB (Walsh, 1959; Leveteau et al., 1971; Mori \& Takagi, 1978). Here, we extend these studies by examining the spatial patterning of this bilateral interaction in response to a functional odorant stimulus in vivo. The bilateral comparison of the information encoded by the paired OBs may support many functions of the olfactory system, including odor localization (Rajan et al., 2006) and discrimination (Cattarelli, 1982).

\section{Centrifugal modulation of the $O B$}

In both turtles and mammals, there are no monosynaptic connections between the paired OBs (Gamble, 1956; Lohman \& Mentink, 1969; Brunjes et al., 2005). Thus, all interbulbar communication must take place via the interaction of retrobulbar structures. The anterior olfactory nucleus has often been studied as a mediator of these interactions due to its extensive bilateral projections to the glomerular layer and granule cells of both OBs (Price \& Powell, 1970; Nakashima et al., 1978; Mori et al., 1979; Luskin \& Price, 1983). However, projections from other cortical areas also excite granule cells (Nakashima et al., 1978), and anterior piriform cortex neurons are sensitive to the lateralization of stimuli from either naris (Wilson, 1997). Furthermore, a number of neuromodulatory systems project to the OB. Cholinergic inputs from the horizontal limb of the diagonal band of Broca have been shown to have mixed effects on mitral, periglomerular and granule cells (Nickell \& Shipley, 1993; Castillo et al., 1999). Noradrenergic projections from the locus coeruleus modulate the reciprocal dendrodendritic mitral cell-granule cell synapse, decreasing the inhibition of mitral cells (Jahr \& Nicoll, 1982). Neuromodulatory systems have been shown to play a critical role in olfactory learning and memory, and computational modeling has suggested that they may increase contrast in mitral cell responses to different odorants (Linster \& Hasselmo, 1997; McLean \& Harley, 2004; Wilson et al., 2004). Centrifugal input to the OB has also been shown to modulate the temporal patterning of $\mathrm{OB}$ activity in a learning-dependent manner (Martin et al., 2004a).

\section{Possible neural substrates for the observed signals}

Centrifugal projections to the $\mathrm{OB}$ target highly specific cell types and laminar regions within the OB structure. Assuming that the voltagesensitive dye stains all membranes equally, the size of the fluorescence signal due to any given neuronal population will be proportional to the average change in membrane potential, the total membrane area of the population and the depth of the population (Zochowski et al., 2000). Several features of the signals described here suggest that they represent the population activity of mitral cells. The long-lasting depolarization observed here and used to quantify OB activation bears a close resemblance in shape and duration to local field potential 
recordings observed in the glomerular layer or EPL, which reverse polarity below the mitral cell layer (Beuerman, 1977). Mitral cells exhibit a long-lasting depolarization, generated in the distal portion of the apical dendrite and propagating to the soma, which is also of similar duration to that observed here (Berkowicz et al., 1994; Carlson et al., 2000). Although voltage-sensitive dye recordings in rodents display a punctuate pattern suggestive of glomerular activation, this pattern is not expected in the recordings made here (Spors \& Grinvald, 2002). The spatial resolution of the photodiode array recordings is $170 \times 170 \mu \mathrm{m}$ per pixel, $\sim 5-10 \times$ the area of a single box turtle glomerulus (Wachowiak et al., 2002). Furthermore, the anatomy of the box turtle OB does not lend itself to resolving single glomeruli. While the glomeruli of the rodent $\mathrm{OB}$ are arranged in a single lamina, the turtle glomerular layer is more than one glomerulus thick and lacks columnar organization, resulting in a more diffuse signal (Wachowiak et al., 2002). This anatomical feature also results in the loss of temporal information in our recordings, so that we cannot discern spatial variations in temporal features of the OB signal that have been observed by others in the activity of ORN input (Spors et al., 2006). Furthermore, the lateral dendrites of mitral cells extend through a large portion of the OB (Shepherd et al., 2004). Thus, the diffuse spatial patterning observed here does not exclude mitral cell activity as a source of the observed signal. In addition, if the observed signal represents activity in mitral cells and especially in the lateral dendrites then the substantial changes in spatial patterning observed here can occur without assuming that changes occur in spatial coding at the level of ORN input to glomeruli.

The interneurons of the $\mathrm{OB}$, and especially granule cells, are expected to be involved in centrifugal control of $\mathrm{OB}$ activity. However, it is unlikely that granule cell activity contributes substantially to the signals described here. Although the interneurons of the $\mathrm{OB}$, and especially granule cells, outnumber mitral cells, the extensive lateral branching of mitral cell dendrites may partially offset this difference (Shepherd et al., 2004). In addition, the scattering of emitted light reduces the voltage sensitive dye signal by $99 \%$ per $\mathrm{mm}$ of tissue, which significantly biases OB recordings toward potential changes in the superficial layers and away from activity in the granule cell layer, the deepest layer of the OB. Furthermore, if granule cell activity constituted a substantial portion of the voltage sensitive dye signal then we would expect paired-pulse stimulation to potentiate, not inhibit, the observed response.

\section{Lateral inhibition and spatial contrast in the $O B$}

Spatial contrast enhancement has been studied in the OB at the levels of both ORN input to the glomerulus and modulation of mitral cell activity in the EPL. Interglomerular centre-surround inhibition has been found to regulate ORN input to the OB both postsynaptically and presynaptically (Aungst et al., 2003; Schafer et al., 2005; Vucinic et al., 2006). Mitral cells are excited when areas of the OB near their apical dendrites are activated, and are inhibited by activation of more distant OB regions (Luo \& Katz, 2001). Contrast-enhancing mechanisms mediated by presynaptic modulation of ORN terminals or interactions between mitral cell dendrites and juxtaglomerular interneurons refine input to the OB on short timescales (Wachowiak et al., 2002; Murphy et al., 2005; Wachowiak et al., 2005).Thus, topographically organized lateral inhibition probably shapes the baseline pattern of $\mathrm{OB}$ activity that is observed here in response to a conditioning odorant stimulus.

While topographically organized inhibition probably plays an important role in $\mathrm{OB}$ function, additional mechanisms may also be at work. There is no a priori relationship between the chemical constituents of a given odorant and the topographic mapping of those constituents within the receptive fields of the OB network. Recent computational studies based on the insect olfactory system have suggested that lateral inhibition among functionally- rather than topographically-related glomeruli effectively enhances spatial contrast in odorant representations (Linster et al., 2005). Further computational work based on the structure of the vertebrate OB has suggested that glomerular circuits can lead to effective nontopographic contrast enhancement without hard-wired interglomerular connections based on response properties (Cleland \& Sethupathy, 2006) These studies suggest that the glomerular layer spatially filters ORN input to the OB, reducing stimulus complexity early in odor processing.

In addition to early filtering of ORN input, OB circuits enhance spatial contrast via the interaction of mitral and granule cells in the EPL. These interactions increase the odorant tuning specificity of individual mitral cells (Yokoi et al., 1995). The EPL receives extensive centrifugal connections, making it well suited for feedback regulation of mitral cell output patterns (Luskin \& Price, 1983; Shepherd et al., 2004). Computational modeling of cholinergic input to the OB suggests that modulation of the mitral cell-granule cell synapse may increase contrast among odorant representations (Linster et al., 2005). Centrifugal modulation of $\mathrm{OB}$ activity may also relieve the topographic constraints of direct interglomerular interaction. Contrast enhancement has been observed in the adaptive responses of olfactory cortex neurons, raising the possibility that the results of cortical computation could feed back to modulate OB responses (Kadohisa \& Wilson, 2006).

\section{Hypothesized role for centrally mediated contrast enhancement}

The time course of inhibition and contrast enhancement we have observed is several seconds, and is consistent with the long-lasting GABAergic inhibition due to dendrodendritic contacts of mitral cells with either periglomerular or granule cells (Mori et al., 1981, 1984; Carlson et al., 2000). The characteristics of the OB response and time course of inhibition suggest modulation of mitral cell activity by contacts in either the glomerular layer or the EPL. However, the heavy projection of commissural anterior olfactory nucleus axons and piriform cortex fibers to granule cells, as well as the projection of neuromodulatory systems to the mitral-granule cell synapse, suggests that the observed contrast enhancement involves mitral-granule cell interactions in the EPL.

The contrast enhancing inhibition that we have observed is consistent with previous results in the turtle olfactory system that suggest olfactory information is encoded by an 'across-fiber' scheme (Pfaffmann, 1959; Erickson, 1963; Wachowiak et al., 2002). In such a scheme, ORNs are broadly tuned and information regarding odorant properties is encoded by the relative activation of glomeruli across the surface of the $\mathrm{OB}$. We thus hypothesize that the response of an unconditioned $\mathrm{OB}$ ipsilateral to a conditioning stimulus reflects the activation of a broad ensemble of glomeruli with varying intensities. Upon the administration of a test stimulus to a conditioned $\mathrm{OB}$, the relative distribution of activity within this group of glomeruli is changed to highlight some features of the response and suppress others. This contrast enhancement could be the result of long-lasting inhibition within intrinsic OB networks, as reflected in the response to an ipsilateral conditioning stimulus. In this case, the information determining the relative inhibition of different $\mathrm{OB}$ regions must be contained exclusively within the initial distribution of glomerular activations or within the functional connectivity of $\mathrm{OB}$ networks. 
Inhibition may also result from centrifugal feedback modulating the activity of granule cells and the strength of mitral-granule cell synapses, a component isolated by the response to a contralateral conditioning stimulus. In this case, the information determining the pattern of inhibition may reside in central olfactory processing regions and may reflect complex processes of pattern recognition and completion. The pattern of inhibition which shapes this contrast enhancement is odorant-specific, as suggested by the result of delivering different odorants for the conditioning and test stimuli. Inhibition is increased because only those $\mathrm{OB}$ regions present in the centrally mediated pattern of both odorants remain activated after inhibition.

\section{Conclusions}

The OB receives heavy centrifugal projections from both olfactory cortical and neuromodulatory regions. We have used a contralateral paired-pulse stimulation paradigm to isolate the influence of feedback from retrobulbar computations from the computation performed in networks intrinsic to the OB. Our data suggest that computations in both retrobulbar and intrinsic networks increase spatial contrast in odorant representations in the OB. Although additional experiments are required to anatomically define the source of contrast enhancement and examine presynaptic and postsynaptic patterning with glomerular resolution, the contralateral paired-pulse stimulation paradigm provides a tool for separating multiple sources of OB modulation in an intact in vivo system. These results provide physiological evidence that, rather than acting as a filter in a feed-forward system, the OB is part of a distributed cortical network which dynamically processes odor information (Haberly, 2001).

\section{Acknowledgements}

This work was supported by a University of Michigan Research Incentives Grant (M.Z.). B.H.S. is supported by the UMMS Medical Scientist Training Program (NIH T32-GM007863) and Hearing, Balance, and Chemical Senses Training Program (NIH T32-DC-00011). We thank Sarah Feldt for assistance with some recordings and Larry Cohen for helpful discussions.

\section{Abbreviations}

$\mathrm{C}_{1}$ and $\mathrm{C}_{2}, 1 \mathrm{~s}$ pulses of odorant selectively delivered (with an ISI) to the naris contralateral to the imaged OB; CPPS, contralateral paired-pulse stimulation; EPL, external plexiform layer; $\mathrm{I}_{1}$ and $\mathrm{I}_{2}, 1 \mathrm{~s}$ pulses of odorant selectively delivered (with an ISI) to the naris ipsilateral to the imaged OB; IPPS, ipsilateral paired-pulse stimulation; ISI, interstimulus interval; OB, olfactory bulb; ORN, olfactory receptor neuron.

\section{References}

Allison, A.C. (1953) The Morphology of the olfactory system in the vertebrates. Biol. Rev. Cambridge Philosoph. Soc., 28, 195-244.

Aungst, J.L., Heyward, P.M., Puche, A.C., Karnup, S.V., Hayar, A., Szabo, G. \& Shipley, M.T. (2003) Centre-surround inhibition among olfactory bulb glomeruli. Nature, 426, 623-629.

Berkowicz, D.A., Trombley, P.Q. \& Shepherd, G.M. (1994) Evidence for glutamate as the olfactory receptor cell neurotransmitter. J. Neurophysiol., 71, 2557-2561.

Beuerman, R.W. (1977) Slow potentials in the turtle olfactory bulb in response to odor stimulation of the nose and electrical stimulation of the olfactory nerve. Brain Res., 128, 429-445.

Brunjes, P.C., Illig, K.R. \& Meyer, E.A. (2005) A field guide to the anterior olfactory nucleus (cortex). Brain Res. Brain Res. Rev., 50, 305-335.

Carlson, G.C., Shipley, M.T. \& Keller, A. (2000) Long-lasting depolarizations in mitral cells of the rat olfactory bulb. J. Neurosci., 20, 2011-2021.
Castillo, P.E., Carleton, A., Vincent, J.D. \& Lledo, P.M. (1999) Multiple and opposing roles of cholinergic transmission in the main olfactory bulb. J. Neurosci., 19, 9180-9191.

Cattarelli, M. (1982) The role of the medial olfactory pathways in olfaction: behavioral and electrophysiological data. Behav. Brain Res., 6, 339-364.

Cleland, T.A. \& Linster, C. (2005) Computation in the olfactory system. Chem. Senses, 30, 801-813.

Cleland, T.A. \& Sethupathy, P. (2006) Non-topographical contrast enhancement in the olfactory bulb. BMC Neurosci., 7, 7.

Cook, P.B. \& McReynolds, J.S. (1998) Lateral inhibition in the inner retina is important for spatial tuning of ganglion cells. Nat. Neurosci., 1 , 714-719.

Davis, R.L. (2004) Olfactory learning. Neuron, 44, 31-48.

Erickson, R.P. (1963) Sensory neural patterns and gustation. In Zotterman, Y. (ed.), Olfaction and Taste. Pergamon, Oxford, pp. 205-213.

Friedrich, R.W. \& Korsching, S.I. (1997) Combinatorial and chemotopic odorant coding in the zebrafish olfactory bulb visualized by optical imaging. Neuron, 18, 737-752.

Gamble, H.J. (1956) An experimental study of the secondary olfactory connexions in testudo-graeca. J. Anat., 90, 15-\&;

Getchell, T.V. (1986) Functional properties of vertebrate olfactory receptor neurons. Physiol. Rev., 66, 772-818.

Grinvald, A., Frostig, R.D., Lieke, E. \& Hildesheim, R. (1988) Optical imaging of neuronal activity. Physiol. Rev., 68, 1285-1366.

Grinvald, A., Lieke, E.E., Frostig, R.D. \& Hildesheim, R. (1994) Cortical point-spread function and long-range lateral interactions revealed by realtime optical imaging of macaque monkey primary visual cortex. J. Neurosci., 14, 2545-2568.

Haberly, L.B. (2001) Parallel-distributed processing in olfactory cortex: new insights from morphological and physiological analysis of neuronal circuitry. Chem. Senses, 26, 551-576.

Jahr, C.E. \& Nicoll, R.A. (1982) Noradrenergic modulation of dendrodendritic inhibition in the olfactory bulb. Nature, 297, 227-229.

Johnson, B.A. \& Leon, M. (2000) Modular representations of odorants in the glomerular layer of the rat olfactory bulb and the effects of stimulus concentration. J. Comp. Neurol., 422, 496-509.

Kadohisa, M. \& Wilson, D.A. (2006) Olfactory cortical adaptation facilitates detection of odors against background. J. Neurophysiol., 95, 1888-1896.

Kauer, J.S. \& Moulton, D.G. (1974) Responses of olfactory bulb neurones to odour stimulation of small nasal areas in the salamander. J. Physiol. (Lond.), 243, 717-737.

Kerr, D. (1960) Properties of the olfactory efferent system. Aust. J. Exp. Biol. Med. Sci., 38, 29-36.

Lam, Y.W., Cohen, L.B., Wachowiak, M. \& Zochowski, M.R. (2000) Odors elicit three different oscillations in the turtle olfactory bulb. J. Neurosci., 20, 749-762.

Lam, Y.W., Cohen, L.B. \& Zochowski, M.R. (2003) Odorant specificity of three oscillations and the DC signal in the turtle olfactory bulb. Eur. J. Neurosci., 17, 436-446.

Leveteau, J., Daval, G. \& MacLeod, P. (1971) The role of the olfactory nucleus in interbulbar inhibition. In Schnider, D. (ed.), Olfaction and Taste IV. Wissenschaftliche Verlagsgesellschaft, Starnberg, pp. 135-141.

Leveteau, J. \& MacLeod, P. (1969) Reciprocal inhibition at glomerular level during bilateral olfactory stimulation. In Pfaffmann, C. (ed.), Olfaction and Taste III. Rockefeller University Press, New York, pp. 212-215.

Linster, C. \& Hasselmo, M. (1997) Modulation of inhibition in a model of olfactory bulb reduces overlap in the neural representation of olfactory stimuli. Behav. Brain Res., 84, 117-127.

Linster, C., Sachse, S. \& Galizia, C.G. (2005) Computational modeling suggests that response properties rather than spatial position determine connectivity between olfactory glomeruli. J. Neurophysiol., 93, 3410-3417.

Lohman, A.H.M. \& Mentink, G.M. (1969) Lateral olfactory tract anterior commissure and cells of olfactory bulb. Brain Res., 12, 396-413.

Luo, M. \& Katz, L.C. (2001) Response correlation maps of neurons in the mammalian olfactory bulb. Neuron, 32, 1165-1179.

Luskin, M.B. \& Price, J.L. (1983) The topographic organization of associational fibers of the olfactory system in the rat, including centrifugal fibers to the olfactory bulb. J. Comp. Neurol., 216, 264-291.

Martin, C., Gervais, R., Hugues, E., Messaoudi, B. \& Ravel, N. (2004a) Learning modulation of odor-induced oscillatory responses in the rat olfactory bulb: a correlate of odor recognition? J. Neurosci., 24, 389-397.

Martin, C., Gervais, R., Chabaud, P., Messaoudi, B. \& Ravel, N. (2004b) Learning-induced modulation of oscillatory activities in the mammalian olfactory system: the role of the centrifugal fibres. J. Physiol. (Paris), 98, $467-478$. 
McLean, J.H. \& Harley, C.W. (2004) Olfactory learning in the rat pup: a model that may permit visualization of a mammalian memory trace. Neuroreport, 15, 1691-1697.

Meister, M. \& Bonhoeffer, T. (2001) Tuning and topography in an odor map on the rat olfactory bulb. J. Neurosci., 21, 1351-1360.

Mori, K., Nagao, H. \& Yoshihara, Y. (1999) The olfactory bulb: coding and processing of odor molecule information. Science, 286, 711-715.

Mori, K., Nowycky, M.C. \& Shepherd, G.M. (1981) Analysis of a longduration inhibitory potential in mitral cells in the isolated turtle olfactory bulb. J. Physiol. (Lond.), 314, 311-320.

Mori, K., Nowycky, M.C. \& Shepherd, G.M. (1984) Synaptic excitatory and inhibitory interactions at distal dendritic sites on mitral cells in the isolated turtle olfactory bulb. J. Neurosci., 4, 2291-2296.

Mori, K., Satou, M. \& Takagi, S.F. (1979) Axonal projection of anterior olfactory nuclear neurons to the olfactory bulb bilaterally. Exp. Neurol., 64, 295-305.

Mori, K. \& Takagi, S.F. (1978) Activation and inhibition of olfactory bulb neurones by anterior commissure volleys in the rabbit. J. Physiol. (Lond.), 279, 589-604.

Murphy, G.J., Darcy, D.P. \& Isaacson, J.S. (2005) Intraglomerular inhibition: signaling mechanisms of an olfactory microcircuit. Nat. Neurosci., 8, 354364.

Nakashima, M., Mori, K. \& Takagi, S.F. (1978) Centrifugal influence on olfactory bulb activity in the rabbit. Brain Res., 154, 301-306.

Nickell, W.T. \& Shipley, M.T. (1993) Evidence for presynaptic inhibition of the olfactory commissural pathway by cholinergic agonists and stimulation of the nucleus of the diagonal band. J. Neuroscience, 13, 650-659.

Pfaffmann, C. (1959) The afferent code for sensory quality. Am. Psychol, 14, 226-237.

Price, J.L. \& Powell, T.P. (1970) An electron-microscopic study of the termination of the afferent fibres to the olfactory bulb from the cerebral hemisphere. J. Cell Sci., 7, 157-187.

Rajan, R., Clement, J.P. \& Bhalla, U.S. (2006) Rats smell in stereo. Science, 311, 666-670.

Ravel, N., Chabaud, P., Martin, C., Gaveau, V., Hugues, E., Tallon-Baudry, C., Bertrand, O. \& Gervais, R. (2003) Olfactory learning modifies the expression of odour-induced oscillatory responses in the gamma $(60-90 \mathrm{~Hz})$ and beta $(15-40 \mathrm{~Hz})$ bands in the rat olfactory bulb. Eur. J. Neurosci., 17, 350-358.

Ross, W.N., Salzberg, B.M., Cohen, L.B., Grinvald, A., Davila, H.V., Waggoner, A.S. \& Wang, C.H. (1977) Changes in absorption, fluorescence, dichroism, and birefringence in stained giant axons: optical measurement of membrane potential. J. Membr. Biol., 33, 141-183.

Rubin, B.D. \& Katz, L.C. (1999) Optical imaging of odorant representations in the mammalian olfactory bulb. Neuron, 23, 499-511.
Schafer, J.R., Kida, I., Rothman, D.L., Hyder, F. \& Xu, F. (2005) Adaptation in the rodent olfactory bulb measured by fMRI. Magn. Reson. Med., 54, 443-448.

Shepherd, G.M., Chen, W.R. \& Greer, C.A. (2004) Olfactory bulb. In Shepherd, G.M. (ed.), Synaptic Organization of the Brain. Oxford University Press, New York, pp. 165-216.

Spors, H. \& Grinvald, A. (2002) Spatio-temporal dynamics of odor representations in the mammalian olfactory bulb. Neuron, 34, 301-315.

Spors, H., Wachowiak, M., Cohen, L.B. \& Friedrich, R.W. (2006) Temporal dynamics and latency patterns of receptor neuron input to the olfactory bulb. J. Neurosci., 26, 1247-1259.

Suga, N. (1995) Sharpening of frequency tuning by inhibition in the central auditory system: tribute to Yasuji Katsuki. Neurosci. Res., 21, 287-299.

Sullivan, S.L., Ressler, K.J. \& Buck, L.B. (1995) Spatial patterning and information coding in the olfactory system. Curr. Opin. Genet. Dev., 5, 516523.

Urban, N.N. (2002) Lateral inhibition in the olfactory bulb and in olfaction. Physiol. Behav., 77, 607-612.

Vucinic, D., Cohen, L.B. \& Kosmidis, E.K. (2006) Interglomerular centersurround inhibition shapes odorant-evoked input to the mouse olfactory bulb in vivo. J. Neurophysiol., 95, 1881-1887.

Wachowiak, M. \& Cohen, L.B. (1999) Presynaptic inhibition of primary olfactory afferents mediated by different mechanisms in lobster and turtle. J. Neurosci., 19, 8808-8817.

Wachowiak, M., Cohen, L.B. \& Zochowski, M.R. (2002) Distributed and concentration-invariant spatial representations of odorants by receptor neuron input to the turtle olfactory bulb. J. Neurophysiol., 87, 1035-1045.

Wachowiak, M., McGann, J.P., Heyward, P.M., Shao, Z., Puche, A.C. \& Shipley, M.T. (2005) Inhibtion of olfactory receptor neuron input to olfactory bulb glomeruli mediated by suppression of presynaptic calcium influx. J. Neurophysiol., 94, 2700-2712.

Walsh, R.R. (1959) Olfactory bulb potentials evoked by electrical stimulation of the contralateral bulb. Am. J. Physiol., 196, 327-329.

Wilson, D.A. (1997) Binaral interactions in the rat piriform cortex. J. Neurophysiol., 78, 160-169.

Wilson, D.A., Fletcher, M.L. \& Sullivan, R.M. (2004) Acetylcholine and olfactory perceptual learning. Learn. Mem., 11, 28-34.

Yokoi, M., Mori, K. \& Nakanishi, S. (1995) Refinement of odor molecule tuning by dendrodendritic synaptic inhibition in the olfactory bulb. Proc. Natl Acad. Sci. USA, 92, 3371-3375.

Zochowski, M.R. \& Cohen, L.B. (2005) Oscillations in the olfactory bulb carry information about odorant history. J Neurophysiol., 94, 2667-2675.

Zochowski, M., Wachowiak, M., Falk, C.X., Cohen, L.B., Lam, Y.W., Antic, S. \& Zecevic, D. (2000) Imaging membrane potential with voltage-sensitive dyes. Biol. Bull., 198, 1-21. 